Gefässchirurgie 2021 · 26:290-293

https://doi.org/10.1007/s00772-021-00746-4

Angenommen: 6. Januar 2021

Online publiziert: 28. Januar 2021

(c) Springer Medizin Verlag GmbH, ein Teil von Springer Nature 2021

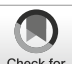

\section{Anamnese}

Ein 49-jähriger Patient mit Schmerzen im linken Fuß sowie fortgeschrittenem sensorischen und leichtem motorischen Defizit stellte sich über die zentrale Notaufnahme vor. Die Beschwerden bestünden seit 4-6h. Der linke Fuß zeigte sich kühl und abgeblasst, mit verzögerter Venenfüllung und verlängerter Rekapillarisierung. Im betroffenen linken Bein ließen sich peripher keine Pulse tasten, rechts bestand kein Pulsdefizit.

\section{Befund}

In einer MR-angiographischen Darstellung der Becken- und Beingefäße zeigte sich ein, auf der Aortenbifurkation, reitender Thrombus mit Ausdehnung in die linke A. iliaca communis. Zudem zeigte sich die linke A. profunda femoris sechs Zentimeter distal ihres Abgangs sowie die Unterschenkelgefäße ab A.-popliteaSegment II thrombotisch verschlossen. Lediglich die A. tibialis anterior kam über Kollateralen am Unterschenkel zur Darstellung. Die rechte Becken-Beinstrombahn imponierte unauffällig (• Abb. 1a). Aufgrund einer Ausbruchssituation mit dem Sars-CoV2-Virus in der Arbeitsstätte des Patienten sowie febriler Temperaturen und trockenen Hustens wurde bereits neun Tage zuvor ambulant ein SARS-CoV-2-Virus-Abstrich durch-

J. Meier · F. A. Vallejos Köhnenkamp · K. Zierke · P.-A. Triebke · H.-G. Fieguth

Thorax- und Gefäßchirurgie, KRH Klinikum Siloah, Hannover, Deutschland

\title{
Reitender Thrombus im Bereich der Aortenbifurkation und kritische Ischämie der linken Beinstrombahn in Zusammenhang mit einer SARS-
CoV-2-Infektion
}

geführt, welcher ein positives Resultat erbrachte. Ein erneuter PCR-Test am Aufnahmetag bestätigte dieses Ergebnis. An Vorerkrankungen wurde ein Lichen planus ruber angegeben, der Patient nahm keine regelmäßigen Medikamente ein.

\section{Diagnose}

Reitender Thrombus in der Aortenbifurkation mit Ausdehnung in die linke A. iliaca communis sowie thromboembolischem Gefäßverschluss der linken Beinstrombahn mit akuter kritischer Extremitätenischämie vor dem Hintergrund einer SARS-CoV-2-Infektion.

\section{Therapie}

Es erfolgte die notfallmäßige Embolektomie mittels Fogarty-Katheter der infrarenalen Aorta über einen offenen transfemoralen Zugang beidseits sowie die Embolektomie der linken A. profunda femoris und der Unterschenkelgefäße. Simultan erfolgte die systemische Applikation unfraktionierten Heparins intravenös mit einer Verlängerung der partiellen Thromboplastinzeit (PTT) auf $>200 \mathrm{sec}$ mit wiederholter Kontrolle nach $30 \mathrm{~min}$. Aufgrund des verzögerten Abstroms im Bereich des Unterschenkels sowie noch verbliebener peripherer Thromben wurde über einen Katheter, welcher im PII-Segment der A. poplitea platziert wurde, $10 \mathrm{mg}$ Actilyse appliziert (- Abb. 1b-d). Die dargestellten Leistengefäße wiesen keine Atheromatose auf. Unmittelbar postoperativ ließ sich ein Puls über der linken A. tibialis posterior tasten, bereits am Folgetag auch kräftig über der linken A. dorsalis pedis.

Als alternatives Verfahren wurde die perkutane Aspirationsthrombektomie in Erwägung gezogen. Aufgrund der hohen Thrombuslast sowie der Gefahr der peripheren Embolisation wurde Abstand von dieser Option genommen und die konventionelle, offenen chirurgische Embolektomie via Fogarty-Katheter favorisiert.

\section{Verlauf}

Postoperativ gelangte der Patient nachbeatmet mit moderaten Beatmungsdrücken mit einem $\mathrm{FiO}_{2}$ von $30 \% \mathrm{O}_{2}$ auf die Intensivstation. In der Blutgasanalyse zeigten sich nahezu Normwerte. Der Patient konnte innerhalb der ersten Stunde nach Eintreffen auf der Intensivstation komplikationslos extubiert werden. Im weiteren Verlauf gelangte der Patient am 1. postoperativen Tag auf die Normalstation, es zeigten sich in der Folge weder respiratorische Komplikationen noch weitere thromboembolische Ereignisse. Der Patient konnte am 6. postoperativen Tag in einem guten Allgemeinzustand, ohne persistierende sensomotorische Einschränkungen der linken un- 

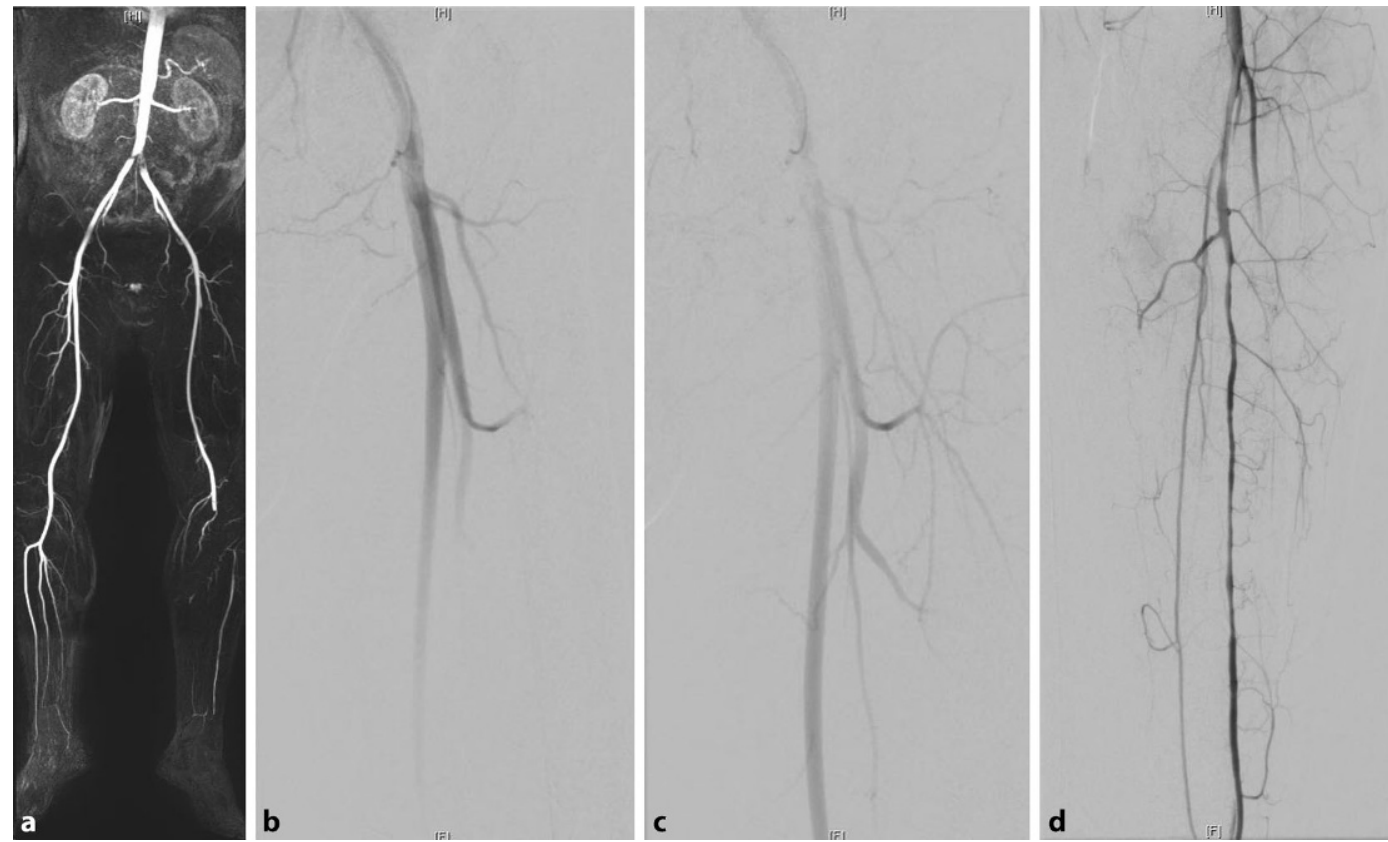

Abb. $1<$ MR-Angiographie der Becken- und Beingefäße sowie intraoperative DSA (Digitale Subtraktionsangiographie). Akuter arterieller Verschluss: Thrombus der Aortenbifurkation und A. iliaca communis sin. sowie der A. femoralis profunda sin. und des Unterschenkels (a). Intraoperative DSA nach erfolgter Embolektomie, fre perfundierte A. femoralis superficialis und $A$. profunda femoris $\sin$. (b, c), 2-Gefäßversorgung am Unterschenkel mit Gefäßspasmus, persistierender embolischer Verschluss der A. tibialis anterior (d)

teren Extremität mit kräftig palpablen Pulsen im Bereich A. tibialis posterior sowie A. dorsalis pedis in die ambulante Betreuung entlassen werden. Postoperativ erfolgte eine Thrombozytenaggregationshemmung mittels Aspirin $100 \mathrm{mg}$ täglich sowie die intravenöse Applikation von unfraktioniertem Heparin im Sinne einer therapeutischen Antikoagulation für zunächst $24 \mathrm{~h}$, im Verlauf Umstellung auf eine prophylaktische Therapie mittels niedermolekularem Heparin und Thrombozytenaggregationshemmung.

Es erfolgte die Empfehlung zur Fortführung der Thrombozytenaggregationshemmung lebenslang sowie zusätzlich die Gabe eines neuen oralen Antikoagulanz (NOAK) für sechs Monate. Darüber hinaus wurde die Empfehlung zur Durchführung einer ambulanten Untersuchung eines Langzeit-Elektrokardiogramms sowie transthorakale bzw. transösophageale Echokardiographie zum Ausschluss einer kardialen Genese des thromboembolischen Ereignisses nach Ablauf der Infektiosität ausgesprochen.

Laborchemisch zeigten sich die $\mathrm{D}$ Dimere direkt postoperativ 17,92-fach erhöht $(8,96 \mathrm{mg} / \mathrm{l} \mathrm{FE})$, am 1 . postoperativen Tag 13,8 -fach erhöht $(6,90 \mathrm{mg} / \mathrm{l}$ $\mathrm{FE})$ und vor Entlassung eine 2,86-fache (1,43 mg/l FE) Erhöhung des Normwertes. Darüber hinaus zeigten sich folgen- de Gerinnungsfaktoren erhöht: Faktor V > $120 \%$ (Norm 70-120\%), Faktor VIII $480 \%$ (Norm 70-150\%), Faktor IX $154 \%$ (Norm 70-120\%) und Faktor X 122\% (Norm 70-120\%) (• Tab. 1).

\section{Diskussion}

Der Zusammenhang zwischen dem neuartigen SARS-CoV-2-Virus und dem Auftreten venöser sowie arterieller thrombotischer Ereignisse ist bereits mehrfach beschrieben worden. Die erhöhte Koagulopathie des Blutes wird hierbei als eigenständiger Risikofaktor für eine erhöhte Morbidität und Mortalität erwähnt. Dazu beitragen könnten die Abnahme des Vasodilatators Angiotensin, der Anstieg des Vasokonstriktors Angiotensin II sowie der, durch die systemische Infektion freigesetzte, Zytokinsturm [1]. Der Angiotensin-ConvertingEnzyme 2(ACE2)-Rezeptor dient dem SARS-CoV-2-Virus als Angriffspunkt, um die Wirtszelle zu infizieren. Der Rezeptor wird unter anderem auf Endothelzellen exprimiert. Anhand histopathologischer Untersuchungen konnten Bestandteile des Virus sowie eine Ansammlung von Entzündungszellen am Endothel nachgewiesen werden. Anhand dieser Beobachtungen beschrieb eine Arbeitsgruppe des Institutes für Pathologie und Molekulare Pathologie Zürich, dass die Infektion mit dem SARS-CoV-2Virus die Ausprägung einer Endothelitis in verschiedenen Organen als direkter viraler Einfluss begünstigen könnte [2]. Bei besonders schweren Krankheitsverläufen wird eine übermäßig stark ablaufende Blutgerinnung sowie disseminierte intravaskuläre Gerinnung (DIC) im Vergleich zu leichteren Verläufen ( $71,4 \%$ vs. $0,6 \%)$ beschrieben [3]. Ein besonderes Augenmerk wird hierbei auf intensivpflichtige Patienten gelegt, bei denen vor allen venös auftretende embolische Ereignisse, trotz Einleitung von thromboseprophylaktischen Maßnahmen, beschrieben werden [4]. In einer großen Fallserie eines Universitätskrankenhauses in Madrid wurden die medizinischen Untersuchungsergebnisse von fast 1500 Patienten analysiert, die positiv auf das SARS-CoV-2-Virus getestet wurden. Eine systemische arterielle Thrombose wurde bei $14 \mathrm{der}$ hospitalisierten Patienten detektiert. Davon wurde bei drei Patienten ein akutes Koronarsyndrom und bei acht Patienten eine cerebrovaskuläre Insuffizienz mit akutem Schlaganfall oder transitorischer ischämischer Attacke beschrieben. Bei drei weiteren Patienten konnte eine Ischämie der unteren infrapoplitealen Extremität gesichert werden, die bei allen drei Patienten mit Nekrosen im Zehenbereich einhergingen, jedoch konservativ 
Der interessante Fall

\begin{tabular}{|c|c|c|c|c|c|c|}
\hline & OP-Tag & 1. post OP Tag & 2. post OP Tag & 3. post OP Tag & 4. post OP Tag & 5. post OP Tag \\
\hline D-Dimere $(<0,5 \mathrm{mg} / \mathrm{I} \mathrm{FE})$ & 8,96 & 6,90 & & & & 1,43 \\
\hline Thrombozyten (Tsd./ $\mu l)$ & 170 & 157 & 193 & 223 & 239 & - \\
\hline $\operatorname{aPTT}(21,6-28,7 \mathrm{sec})$. & & 30,8 & $<20,0$ & & $<20,0$ & $<20,0$ \\
\hline C3-Komplement $(0,9-1,8 \mathrm{~g} / \mathrm{l})$ & n.u. & n.u. & n.u. & 1,17 & n.u. & n.u. \\
\hline C4-Komplement $(0,1-0,4 \mathrm{~g} / \mathrm{l})$ & n.u. & n.u. & n.u. & 0,20 & n.u. & n.u. \\
\hline Faktor II (70-120\%) & n.u. & n.u. & n.u. & n.u. & n.u. & 116 \\
\hline Faktor V (70-120\%) & n.u. & n.u. & n.u. & n.u. & n.u. & $>120$ \\
\hline Faktor VII (70-120\%) & n.u. & n.u. & n.u. & n.u. & n.u. & 120 \\
\hline Faktor VIII (70-150\%) & n.u. & n. u. & n. u. & n.u. & n.u. & 480 \\
\hline Faktor IX (70-120\%) & n.u. & n.u. & n.u. & n.u. & n.u. & 154 \\
\hline Faktor X (70-120\%) & n.u. & n.u. & n.u. & n.u. & n.u. & 122 \\
\hline Faktor XII (70-150\%) & n.u. & n.u. & n.u. & n.u. & n.u. & 102 \\
\hline Faktor XIII (70-140\%) & n.u. & n.u. & n.u. & n.u. & n.u. & 74 \\
\hline Rheumafaktoren $(<14 \mathrm{IU} / \mathrm{ml})$ & n.u. & n.u. & n.u. & 9 & n.u. & n.u. \\
\hline
\end{tabular}

behandelt wurden. Die Mortalitätsrate von Patienten, die positiv auf SARSCoV-2-Virus getestet wurden und bei denen eine arterielle Ischämie detektiert wurde, lag bei $28,6 \%$. Gemäß der patientenbezogenen Daten nahmen vier Patienten ein Medikament zur Thrombozytenaggregationshemmung ein, bei zwei Patienten bestand eine orale Antikoagulation. Von den Patienten mit dem Nachweis eines arteriellen thrombotischen Ereignisses erhielten $70 \%$ eine Thromboseprophylaxe mittels Heparin, jeweils zur Hälfte in therapeutischer und prophylaktischer Dosierung [5].

Der zeitliche Zusammenhang von positivem Nachweis der SARS-CoV-2Infektion und dem Auftreten arterieller thrombotischer Ereignisse lässt den Schluss zu, dass in den ersten drei Wochen, mit einem Maximum in der zweiten Woche, eine erhöhte Rate vorliegt [6]. Es liegen bereits aktualisierte Empfehlungen zur Thromboseprophylaxe für SARS-CoV-2-Infektion von der deutschen Gesellschaft für Thrombose- und Hämostaseforschung e.V. (GTH) vor, die eine kontinuierliche Überprüfung der Indikation einer medikamentösen Thromboseprophylaxe mittels niedermolekularem Heparin zur Vermeidung venöser Thromboembolien (VTE) empfiehlt. Hierbei wird nicht zwischen hospitalisierten und ambulanten Patienten unterschieden. Darüber hinaus empfiehlt die GTH bei symptomatischen
Patienten die Bestimmung der D-Dimere. Bei Vorliegen zusätzlicher Risikofaktoren (BMI $>30 \mathrm{~kg} / \mathrm{m}^{2}$, stattgehabte VTE, aktive Krebserkrankung) oder intensivstationärer Behandlung wird eine intensivierte Thromboseprophylaxe in halbtherapeutischer Dosierung empfohlen, in Abhängigkeit der Nierenfunktion und des Blutungsrisikos. Darüber hinaus wird ein kontinuierliches Monitoring der hämostaseologischen Parameter bei hospitalisierten Patienten empfohlen, bei fortbestehender Immobilität, hoher entzündlicher Aktivität oder dem Vorhandensein zusätzlicher Risikofaktoren wird empfohlen, die Indikation zur Fortführung einer ambulanten Thromboseprophylaxe zu prüfen [7]. In Anlehnung an die Empfehlungen der GTH wäre bei unserem Patienten die Bestimmung der D-Dimere aufgrund der subfebrilen Temperatur und des Hustens bereits vor Auftreten des embolischen Ereignisses sinnvoll gewesen. Erhöhte D-Dimere wiederum hätten eine Thromboseprophylaxe zur Konsequenz gehabt. Das operative Vorgehen entsprach den Leitlinien bei Patienten mit Embolien anderer Genese. Festzuhalten bleibt das gute Ansprechen auf die intraarterielle Lysetherapie. So waren die restlichen verbliebenen Thromben in der A. tibialis anterior, die in der intraoperativen DSA (Digitale Subtraktionsangiographie) nach Fogarty-Manöver zur Darstellung kamen, in der sonographischen Verlaufskontrolle am 4. postoperativen Tag nicht mehr nachweisbar.

In der vorliegenden Anamnese wird ein Lichen planus ruber erwähnt, der sich in der histopathologischen Analyse charakteristisch mit einer akanthotisch verbreiterten Epidermis zeigte. Wechselnd wurde eine ausgeprägte Hypergranulose und subepidermale bandförmige lichenoide lymphozytäre Entzündungsreaktion beschrieben. Die Basalmembran zeigte sich stellenweise aufgesplittert und von Lymphozyten überlagert. Diese Beschreibungen sind mit einem Lichen planus ruber vereinbar [8]. Obwohl die Ätiologie bisher nicht vollständig geklärt ist, weisen verschiedene Studien auf eine immunologische Pathogenese hin. Lymphozyten, im Besonderen T-Zellen sowie antigenpräsentierende Zellen als auch Adhäsionsmoleküle und inflammatorische $\mathrm{Zy}$ tokine werden in diesem Zusammenhang erwähnt [9-11]. Aufgrund von Veränderungen des Blutflusses sowie Oberflächendefekten im Gefäßbett kann im Verlauf die Plättchenaggregation und Gerinnungskaskade initiiert werden [12]. Eine arterielle Thrombose kann in der Folge entstehen.

Der histopathologische Nachweis des Lichen planus ruber wurde bereits 2009 geführt, sodass aufgrund der langen Latenz ein Zusammenhang zum vorliegenden arteriellen embolischen Ereignis als unwahrscheinlich erachtet werden kann. 


\section{Fazit für die Praxis}

Zusammenfassend wird empfohlen, dass hospitalisierte Patienten mit einer SARS-CoV-2-Infektion eine Thromboseprophylaxe erhalten sollten, sofern keine Kontraindikationen vorliegen. Die Bestimmung von d-Dimeren ist sinnvoll, um die Indikation einer Thromboseprophylaxe bei signifikant erhöhten Werten zu bekräftigen. Patienten mit positivem SARS-CoV-2-Virusnachweis und kritischer arterieller Ischämie sind mit konventionellen gefäßchirurgischen Methoden therapierbar.

\section{Korrespondenzadresse}

\section{Prof. Dr. H.-G. Fieguth}

Thorax- und Gefäßchirurgie, KRH Klinikum Siloah

Stadionbrücke 4, 30459 Hannover, Deutschland karin.guenther@krh.de

Interessenkonflikt. J. Meier, F.A. Vallejos Köhnenkamp, K. Zierke, P.-A. Triebke und H.-G. Fieguth geben an, dass kein Interessenkonflikt besteht.

Für diesen Beitrag wurden von den Autoren keine Studien an Menschen oder Tieren durchgeführt. Für die aufgeführten Studien gelten die jeweils dort angegebenen ethischen Richtlinien. Für Bildmaterial oder anderweitige Angaben innerhalb des Manuskripts, über die Patienten zu identifizieren sind, liegt von ihnen und/oder ihren gesetzlichen Vertretern eine schriftliche Einwilligung vor.

\section{Literatur}

1. Tay MZ, Poh CM, Rénia L, MacAry PA, Ng LFP (2020) The trinity of COVID-19: immunity, inflammation and intervention. Nat Rev Immunol 20(6):363-374. https://doi.org/10.1038/s41577-020-0311-8

2. Varga Z, Flammer AJ, Steiger $P$ et al (2020) Endothelial cell infection and endotheliitis in COVID-19. Lancet 395(10234):1417-1418. https:// doi.org/10.1016/S0140-6736(20)30937-5

3. Arachchillage DRJ, Laffan M (2020) Abnormal coagulation parameters are associated with poor prognosis in patients with novel coronavirus pneumonia. J Thromb Haemost 18(5):1233-1234. https://doi.org/10.1111/jth.14820

4. Klok FA, Kruip MJHA, van der Meer NJM et al (2020) Incidence of thrombotic complications in criticallyill ICU patients with COVID-19. Thromb Res 191:145-147. https://doi.org/10.1016/j.thromres. 2020.04.013

5. Cantador E, Núñez A, Sobrino P et al (2020) Incidence and consequences of systemic arterial thrombotic events in COVID-19 patients. J Thromb Thrombolysis. https://doi.org/10.1007/s11239020-02176-7

6. Thachil J, Tang N, Gando S et al (2020) ISTH interim guidance on recognition and management of coagulopathy in COVID-19. J Thromb Haemost
18(5):1023-1026. https://doi.org/10.1111/jth 14810

7. Oldenburg J, Klamtozh R, Langer F (2020) Aktualisierte Empfehlung zur Thromboseprohphylaxe bei SARS-CoV-2 (COVID-19). http://gth-online. org/wp-content/uploads/2020/04/AktualisierteGTH-Empfehlungen-COVID-19-1.pdf. Zugegriffen:26. Juli 2020

8. Lever WF (1997) Histopathology of the skin. In: Elder DE (Hrsg) Lever's histopathology of the skin 8. Aufl. Lippincott-Raven, Philadelphia

9. Boyd AS, Neldner KH (1991) Lichen planus. J Am Acad Dermatol 25:593-619

10. Freedberg IM, Fitzpatrick TB (1999) Fitzpatrick's dermatology in general medicine, 5. Aufl. McGrawHill, New York

11. Porter SR, Kirby A, Olsen I, Barrett W (1997) Immunologic aspects of dermal and oral lichen planus: a review. Oral Surg Oral Med Oral Pathol Oral Radiol Endod 83:358-366

12. Stary HC, Chandler AB, Dinsmore RF et al (1995) A definition of advanced types of atherosclerotic lesions and s histological classification of atherosclerosis: a report from the committee on vascula lesions of the council on Athersclerosis, American heart association. Circulation 92:1355-1374
Evidenz in der minimalinvasiven onkologischen Chirurgie

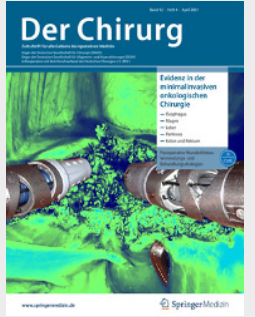

Die Einführung minimalinvasiver Techniken hat wie kaum eine andere Methode die operativen Eingriffe in der Allgemein- und Viszeralchirurgie revolutioniert. Der Chirurg 04/2021 bietet Ihnen Aktuelles zur Evidenz solcher Verfahren in der onkologischen Chirurgie.

Bei der Auswahl der Autor*innen wurde besonders darauf geachtet, dass deren Arbeitsgruppen die genannten Methoden täglich in der eigenen Praxis anwenden. Damit ist gewährleistet, dass nicht nur aktuelle Literaturdaten, sondern auch individuelle Erfahrungen eingebracht wurden. Nutzen Sie die Orientierungsmöglichkeiten für die Wahl des Operationsverfahrens. Verschaffen Sie sich einen aktuellen Überblick über den Einsatz minimalinvasiver OPTechniken in der onkologischen Chirurgie für

$$
\begin{aligned}
& \text { - Ösophagus } \\
& \text { - Magen } \\
& \text { - Leber } \\
& \text { - Pankreas } \\
& \text { - Kolon } \\
& \text { - Rektum }
\end{aligned}
$$

Suchen Sie noch mehr zum Thema? Mit e.Med - den maßgeschneiderten Fortbildungsabos von Springer Medizin - haben Sie Zugriff auf alle Inhalte von SpringerMedizin.de. Sie können schnell und komfortabel in den für Sie relevanten Zeitschriften recherchieren und auf alle Inhalte im Volltext zugreifen.

Weitere Infos zu e.Med finden Sie auf springermedizin.de unter "Abos" 